\title{
Spermatid Retention
}

National Cancer Institute

\section{Source}

National Cancer Institute. Spermatid Retention. NCI Thesaurus. Code C120908.

Persistence of mature elong ating spermatids in the seminiferous tubules after the normal stage of physiologic release. 